not to be accredited as the main cause of death in this case; for the peritonitis was neither intense nor widely spread, nor, during life, more marked than $I$ have seen it in cases that have been freed from it and going on well in a few days; and that in whatever degree the peritonitis may be thought to have been tributary, the main cause lay in the excitability of a sensorium imperfectly constituted, and uuable to bear the shock of the lengthened examination, operation, and chloroform action. I regret, however, now that, in ignorance of the peculiarity of the family history, I did not avail myself of a suggestion made by Mr. Benfield to postpone the operation for a few days.

But if the explanation I have suggested of the sound which was heard by all, though not conclusively for operation, were the right one, the symptoms still remain as a mystery. We occasionally find cases of great pain and frequent micturition of small quantities, and which are attributable to irritable bladder or acrid urine; but these symptoms are usually accompanied by mucous or mucopurulent deposit, which we had not; and never, as far as I know, by sudden stoppage of the stream followed by the passing of a larger quantity immediately after. How far we may accept the explanation ingeniously offered by Mr. Charles W. Wood of Woodhouse Eaves in this county, at the post mortem examination, I leave others to decide. He takes the very dilated state and pouchy form of the ureters as the cause of their more than usually oblique and valvular entrance into the bladder, and of a difficult, intermitting, and painful evacuation of their contents into it, the pain being trans ferred along the canal to the usual site at the glans penis. Certainly, the immense size and irregular form of these conduits were very striking.

\section{CANCER OF THE FEMALE BREAST, WITH ULCERATION.}

OPERATION : LONG IMMUNITY FROM RECURRENCE OF THE DISEASE.

By H. Haynes Walton, F.R.C.S., Surgeon to St. Mary's Hospital, and to the Central London Ophthalrnic Hospital.

[Read before the Medical Society of London.]

THE surgical features in this instance of cancer bear so particularly on the question of operating in a late stage of the affection, that I have determined to give publicity to them, accompanied by an expression of the motives that induced me to act. From them is to be gathered a fact of significance, which may be gladly known, by some, at least, of those who may happen to glance over the report.

In May 185\%, Mr. Harding of Percy Street called me to consult on a lady, 64 years old, who had borne several children, with an ulcerated breast. The ulcer was as large as a shilling. The brief history is, that she discovered a lump in the breast in consequence of pain; but although the painful symptoms increased, she did not apply to Mr. Harding till December 1856. Now, however, she was in great suffering, could not sleep but at very short intervals, and scarcely got relief from any treatment. Ulceration quickly ensued.

Of the malignancy of the disease there could be no doubt; that we were looking on hard cancer, was equally certain; and withal, I determined to operate.

A proper explanation of the circumstances that influenced my decision is all important, because it is one of the standing rules respecting the surgical treatment of cancer, and one, perbaps, on which more stress is usually laid than any other in discussing the propriety of an operation, to abandon all idea of excision when the skin is once broken. I have seen this state alone no few times in private consultations, and still more often in public, made the cause for the immediate and $\zeta$ emphatic abandonment of any operative proceeding. $\mathbb{\$}$ Usually, the opinion is as quickly expressed, as it is decisive.

These were my pleas for operating. There was the $\vec{F}$ paramount indication to check that degree of anguish $\stackrel{\oplus}{+}$ which supervenes only when the skin is involved, and which often induced an expression of a desire for death. $\bar{\sigma}$ There was an absence of all coincident unfavourable $\overline{\bar{\omega}}$ states. As regards the cancer itself, it was evidently in $\frac{\vec{\sigma}}{\Phi}$ the gland-tissue, which seemed but partially involved, ¿ not wholly infiltrated, and inadherent to the wall of the chest. The skin around the ulceration was healthy. The os ulceration was limited, and not rapidly extending.

Respecting the constitutional implication, there really was none; no cachexy; no well marked debility; no $\vec{\omega}$ lymphatic gland implication; no evidence of chest, or $\mathscr{\mathcal { W }}$ of abdominal disease. For all that I could see or dis- 0 cover, there was nothing beyond the local affection.

It would appear to me, that there can be no more suffi- iv cient reason against my practice in such a case than if $G$ there had been no ulceration. Although, when cancer 0 ulcerates, its distructive effect on life would seem then to be nearer, relief from pain is substantial, and not the $\mathrm{N}$ less important to the individual ; and an operation done 응 for that purpose has the strong recommendations of $\vec{J}$ being definite and appropriate. The presence of any $\vec{A}$ one of the symptoms to which $I$ have alluded as being absent, might, of course, in itself, have been objection- $\mathbb{D}$ able to interference by practical surgery.

I removed the whole of the mamma. But this ex- $\frac{\mathbb{}}{3}$ pression may convey more than I intend, except I add that, in all cases of cancer connected with the breast, $\stackrel{\mathbb{D}}{-}$ whether as a palpable infiltration, or apparently more or $\vec{\infty}$ less isolated about the border, I hold it wise to extirpate $\infty$ the entire gland.

The great justification of my proceeding, the unques tionable propriety of it, is to be found unerringly in the result.

My patient derived comfort from the day of the oper: ation, and she was soon about. In a few weeks she called on me at my house. She was quickly restored to $\frac{O}{D}$ health, and her wontedl high animal spirits returned; $\varrho$ she fully enjoyed all the pleasures of life, and pos- $\overrightarrow{\vec{O}}$ sessed a degree of bodily vigour that is usually not met $\frac{0}{3}$ with at her age; was equal to an amount of activity and exercise that few women ten jears younger can accomplish. I saw her a few times full of hope, until the? 14th of October of this year, when she called to show me a lump in the axilla, and to narrate her sufferings. Secondary deposit of cancer had set in. Darting pains in the tumour and along the arm, increasing in severity, induced her to seek any treatment likely to relieve her. 3 As she was to all appearance in better health than when I first saw her, I proposed to remove the cancerous mass, 윽 and fully explained, however, that any benefit must only be regarded as very temporary, and otherwise spoke 응 plainly of the nature of her condition. She consented. With the assistance of Mr. Harding and Mr. Hulme, IN operated. The diseased mass was very deep ; and care- $D$ ful dissection was needed to remove the whole in one piece. I effected my object by picking, scratching, 三. and tearing through the surrounding tissue, rather than $N$ by cutting; and so well was I able to do this, that only N one ligature was required, ard that was for a small tegu- $\omega$ mentary artery. No venous hæmorrhage supervened. The wound, however, was not brought together for four 0 hours. The tumour was of about the size of a large hen's egg. It was made up of several glands, more or less $\Phi$ infiltrated with cancer. Primary adhesion followed; and in ten days, the lady was again out of her room, without pain or discomfort.

If $I$ am acquainted with the final issue of this very in- $\frac{\mathbb{D}}{\mathbb{D}}$ teresting triumph of our surgical art-for surely this is not too strong an expression, when life has been so long 
held under such pleasant circumstances, I shall notice it, with a very short allusion to the antecedent.

That some surgeons would operate in such a case as the above, I am well aware; but, most assuredly, a very large majority would not; and I beg to say, as a last remark, that I am not attempting to lay down any new doctrine, but merely desire to draw more general atten. tion to this one particular, that very undoubted, considerable, and peculiar advantages, may be conferred by an operation on the victim of cancer, even after the ul. cerative stage is established.

69 , Brook Street, Hanover Square, W.

\section{ON THE ACTION OF THE BRONCHIAL MUSCLES.}

By C. Radclyffe Hall, M.D., F.R.C.P., Physician to the Hospital for Consumption, Torquay; etc.

THE perusal in the Medical Times and Gazette of Dr. Hyde Salter's interesting criticism on Dr. Kidd's view of the pathology of asthma, tempts me to say a word in defence of that hypothesis concerning the action of the bronchial muscles, which they both alike reject.

Dr. Kidd considers that the bronchial muscles are inspiratory, and that they cause contraction of the tubes during ordinary inspiration.

Dr. Hyde Salter considers them not "muscles of respiration at all, either of expiration or inspiration."

Haller, Reisseissen, Elliotson, and others, suppose that the bronchial muscles act as auxiliary agents in ordinary expiration. Dr. C. J. B. Williams appears to be of the same opinion. In the Transactions of the British Medical Association for 1850 (p. 363), I published a paper, in which $I$ endeavoured by experiments to substantiate this view. And, unless I remember inaccurately his remarks in conversation, Dr. Brown-Séquard has come to the same conclusion.

There are then three conjectures at present entertained respecting the function of the bronchial muscles.

1. That they contract in ordinary inspiration.

2 . That they contract in ordinary expiration.

3. That they contract only occasionally to expel irritating matters, or in answer to morbid sensations, either in the quick manner of cough, or in the persistent manner of spasm.

This last proposition, apart from its exclusiveness, few will be disposed to deny. Dr. Hyde Salter has excellently well argued that spasm of the bronchial muscles is the sine qua non of true asthma; is indeed the only explanation that will fully work the problem of the disease.

When on each contraction of the heart a wave of blood distends a small artery, the elastic and muscular coats of the blood-vessel yield, and the vessel expands. This answers to inspiration; excepting that, in the case of the circulation, the motor force is from the centre; in that of the respiration, from the circumference. As regards the artery and the bronchial tube, this difference may for the moment be left out of consideration. Immediately after its distension, the artery slightly contracts, and by so doing quickens the onward flow of its contents. This answers to expiration. Galvanise the blood-vessels in a suitable manner, and the small arteries contract. Or, without that, expose the skin to cold, or the mouth to ice, and the same will happen.* This tonic contraction of the blood-vessels answers to asthma.

Hence, the view I am contending for assumes that the bronchial muscles contract slightly for every expira-

* A possibility to be remembered in directing ice to be sucked continuously when the patient is of feeble vital power. Twice in the last year I have seen viceration of the tongue, or of the inside of the cheeks or lips, attributable to this. The ice constringed the bloodvessels so considerably and so long as to induce molecular deathulceration. tion; contract more vigorously and suddenly for every cough; and contract in the persistent spasmodic man. ner in every case of asthma.

The larynx and glottis expand for inspiration, and contract for expiration. The glottis can contract vigorously and suddenly, as in cough; or in the shape of spasm of more or less duration, in laryngismus, in hooping-cough, and in croup. Laryngismus may be considered as an infantile laryngeal asthma. I assume that the remainder of the respiratory tubing in all these respects follows suit.

The smallest bronchial tubes are the most muscular. The larger tubes have the most of those elastic-spring openers, the bronchial cartilages. Hence, the bronchial muscles will mainly operate in the tubes which lie nearest to the finer and more delicate parts of the lung, the vestibular passages and air-cells.

We will assume that the ordinary excitor to action of the bronchial muscles is the disterision of the tube by inspired air. This will take place just after the end of inspiration, when the newly arrived air has undergone expansion by warmth; that is, provided the in-rush be gentle and gradual, as in quiet healthy breathing. But in proportion as the in-rush is sudden, abrupt, and forcible, as when a person is out of breath from running, will the bronchial muscles act vigorously, not to promote inspiration, but to oppose it, and so prevent it from inducing more than a certain safe amount of distension of tubes.

In expiration, the compressed air-cells expel a portion of their air with a given force. The expulsion will be quickened by lessening the bore of the exit channel, provided the expelling force remain adequate. And this quickening of exit will take place most precisely where it is most necessary to get rid of foul air speedily ; viz., in the smallest bronchial tubes which are nearest to the air-cells; less and less as the tubes become larger, and intermixture of foul with fresh air readier; for it must be remembered that, at the moment when expiration commences in the periphery of the lungs, the larger tubes are still full of unused air.

The objection usually urged against the supposition that the bronchial muscles assist in the act of expiration is, that the contraction of a bronchial tube would oppose, and not facilitate the passage of air out, just as it does the passage of air in. This would be so, provided the bore of the tube were lessened out of proportion to the motor force, and provided it were kept persistently in the contracted condition. Within suitable limits, by lessening bore, rate is quickened and volume lessened. And so long as the proper balance is preserved between the quickening effect of lessened calibre of bronchial tube and the amount of propelling power from behind in expiration, we may assume that as much may be gained by increase of speed as is lost by diminution of channel. If the objection were valid, it would be equally strong against the case of the glottis, or that of the artery. Contraction of the glottis must impede both the ingoing and the outgoing of the air, and does so in various morbid conditions. Yet, in the normal state of things, the glottis does expand for inspiration and contract for expiration.

The same person using the spirometer finds that he cannot do full justice to his vital capacity, if he make his trial expiration too quickly, or too slowly. He is stopped by positive inability to do more of the act of expiration, and not by the sensation of apnœa. When too quick, he finishes before he has got out all the expiratory air from the lungs, which he could by a quieter attempt expel. When too slow, he tires the expiratory machinery before he has emptied the lungs of all but the necessary residual air. I suspect that the bronchial muscles are a good deal concerned in occasioning the inadequate performance of these spirometric expirations. The latter portion of the expiration consists in 